\title{
Experimental evaluation of ecological principles to understand and modulate the outcome of bacterial strain competition in gut microbiomes
}

\author{
Rafael R. Segura Munoz ${ }^{1,2}$, Sara Mantz ${ }^{1}$, Ines Martínez ${ }^{3,4}$, Fuyong Li ${ }^{3,5}$, Robert J. Schmaltz ${ }^{1}$, Nicholas A. Pudlo ${ }^{6}$, Karthik Urs ${ }^{6}$, \\ Eric C. Martens (iD ${ }^{6}$, Jens Walter (iD ${ }^{3,4,7 凶}$ and Amanda E. Ramer-Tait (D) ${ }^{1,2 \bowtie}$
}

(c) The Author(s) 2022, corrected publication 2022

\begin{abstract}
It is unclear if coexistence theory can be applied to gut microbiomes to understand their characteristics and modulate their composition. Through experiments in gnotobiotic mice with complex microbiomes, we demonstrated that strains of $A k k e r m a n s i a$ muciniphila and Bacteroides vulgatus could only be established if microbiomes were devoid of these species. Strains of $A$. muciniphila showed strict competitive exclusion, while $B$. vulgatus strains coexisted but populations were still influenced by competitive interactions. These differences in competitive behavior were reflective of genomic variation within the two species, indicating considerable niche overlap for $A$. muciniphila strains and a broader niche space for $B$. vulgatus strains. Priority effects were detected for both species as strains' competitive fitness increased when colonizing first, which resulted in stable persistence of the A. muciniphila strain colonizing first and competitive exclusion of the strain arriving second. Based on these observations, we devised a subtractive strategy for A. muciniphila using antibiotics and showed that a strain from an assembled community can be stably replaced by another strain. By demonstrating that competitive outcomes in gut ecosystems depend on niche differences and are historically contingent, our study provides novel information to explain the ecological characteristics of gut microbiomes and a basis for their modulation.
\end{abstract}

The ISME Journal (2022) 16:1594-1604; https://doi.org/10.1038/s41396-022-01208-9

\section{INTRODUCTION}

The gut microbiota is considered an important aspect of host health, influencing digestion, immune system development, and pathogen invasion [1-7]. Moreover, numerous studies have documented differences in microbiome composition and function between healthy and diseased humans and animals [8, 9]. Strategies aimed at modulating and restoring the ecological and physiological features of the gut microbiome have therefore gained much momentum [10,11]. Considering the complexity of the gut ecosystem, successful modulation of gut microbiomes is likely to require the application of ecological theory [12-14].

The introduction of live microbes, either as single strains (e.g., probiotics, live biotherapeutics) or complex mixtures (e.g., fecal microbiota transplants), into the gut ecosystem represents one approach to modifying the microbiome. However, the ecological requirements for sustained long-term colonization (i.e., engraftment) of orally administered live microbial products are poorly understood [15]. Recent evidence suggests that engraftment may depend on the pre-treatment microbiome composition, especially the absence of closely related species [16-19]. We [17, 18] and others $[16,20-22]$ have shown that the resident microbiome influences engraftment of incoming species, likely through competitive exclusion where newly-arriving species cannot coexist with established species if they occupy exactly the same niche (and are competing for identical resources) [23]. In particular, Maldonado and colleagues showed that persistence of the probiotic Bifidobacterium longum AH1206 in select study participants was associated with low abundance of $B$. longum species [17]. Lack of colonization in other participants may thus be explained by competitive exclusion [23]. However, these findings are based on associations, and it has not been established if competitive exclusion is in fact the causative factor that determines engraftment. Indeed, not all outcomes from microbiome-based interventions are consistent with competitive exclusion and instead indicate coexistence of related strains in fecal transplants $[20,24]$. These discrepancies illustrate that the ecological factors governing engraftment are complex, insufficiently understood, and likely extend beyond the competitive exclusion principle [13].

Modern coexistence theory suggests that competitive exclusion and coexistence of species or strains are determined by equalizing mechanisms that reduce fitness differences among members and

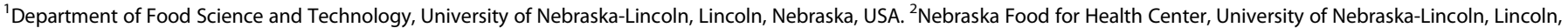

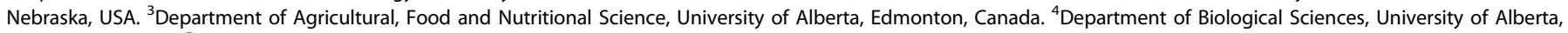

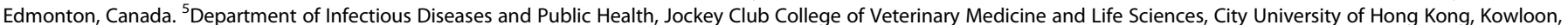

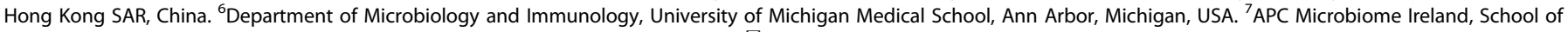

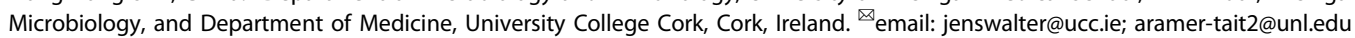


stabilizing mechanisms that decrease competition through niche differences (i.e., resource partitioning) [25, 26]. Competitive interactions are further historically contingent, meaning that order of arrival of a species into an ecosystem can result in priority effects that alter the outcome of species interactions (e.g., by benefiting early colonizers over late colonizers) [26]. For gut ecosystems, competitive exclusion has been demonstrated for isogenic bacterial strains orally administered to germ-free mice $[27,28]$. However, such an experiment represents an extreme condition where the competing strains are essentially identical and have maximum niche overlap. Strains in natural communities possess genetic and trait variation, and strains used as live microbial products are likely to differ from resident microbiota members. To what degree contemporary niche and coexistence theory applies to interactions among members of the gut microbiota and the introduction of microbes into gut microbiomes has not been experimentally tested.

Here, we performed systematic experiments in gnotobiotic mice to test the applicability of coexistence theory to the stable establishment of gut microbes and to determine if such information can be utilized to stably modulate the gut microbiota at the strain level. We selected two species of gut bacteria, Akkermansia muciniphila and Bacteroides vulgatus, based on their importance in modulating host metabolism $[29,30]$ and immunity [31-33]. To study niche occupancy and intraspecific competition under close-to-natural but strictly controlled conditions, we utilized gnotobiotic mice colonized with complex microbiomes with and without A. muciniphila and $B$. vulgatus. We specifically tested (i) to what degree the absence of a species determines colonization, (ii) if colonization can be prevented or altered by prior introduction of another strain of a species, and (iii) the effect of colonization order on coexistence between strains of the same species. Finally, we applied the information gained from these experiments to design a subtractive antibiotic strategy with the aim of removing an $A$. muciniphila strain from an assembled community and replacing it with a different strain.

\section{MATERIALS AND METHODS} Bacteria

Bacterial strains used in this study were: Akkermansia muciniphila BAA-835 (American Tissue Culture Collection, Manassas, VA) isolated from human feces, A. muciniphila YL44 (Leibniz Institute DSMZ, Braunschweig, Germany) isolated from C57BL/6 J mouse feces, Bacteroides vulgatus ATCC 8482 (American Tissue Culture Collection), and B. vulgatus RJ2H1 [18]. A. muciniphila strains were cultured in $10 \mathrm{~mL}$ Brain Heart Infusion (BHI) medium (BD, Sparks, MD) supplemented with $0.3 \%(\mathrm{w} / \mathrm{v})$ mucin from porcine stomach Type II (Sigma-Aldrich, St. Louis, MO) and 0.5\% yeast extract (BD, Sparks, MD); this medium is referred to as BHIm in this manuscript. B. vulgatus strains were grown in $10 \mathrm{~mL}$ BHI medium supplemented with menadione $(0.001 \mathrm{~g} / \mathrm{L}$, Sigma-Aldrich), hemin $(0.005$ $\mathrm{g} / \mathrm{L}$, Sigma-Aldrich), and an additional $0.5 \%$ yeast extract (BD, Sparks, MD); this medium is referred to as BHIs throughout this manuscript. Carbohydrate growth arrays for B. vulgatus 8482 and $\mathrm{RJ} 2 \mathrm{H} 1$ were performed as previously described $[34,35]$.

To inoculate mice, one vial of each bacterial strain was retrieved from $-80^{\circ} \mathrm{C}$ storage and the entire contents were struck onto a plate containing BHIm with agar $(1 \% \mathrm{w} / \mathrm{v})$. Plates were incubated at $37^{\circ} \mathrm{C}$ under anaerobic conditions for either three days for $B$. vulgatus strains or five days for A. muciniphila strains. Colonies were then transferred into $30 \mathrm{~mL} \mathrm{BHIm}$ or BHls and incubated anaerobically at $37^{\circ} \mathrm{C}$. After $24 \mathrm{hr}, 300 \mu \mathrm{L}$ of each culture was transferred into a fresh $30 \mathrm{~mL}$ of BHIm or BHIs. After $18 \mathrm{~h}$, these cultures were centrifuged ( $3000 \times \mathrm{g}$ for $15 \mathrm{~min}$ ), resuspended in 1X PBS (HyClone, Logan, UT) to achieve a concentration of $1.0 \times 10^{9} \mathrm{CFU} / \mathrm{mL}$, and administered to mice via oral gavage $(100 \mu \mathrm{L} /$ mouse). For some experiments, A. muciniphila BAA-835 and B. vulgatus ATCC 8482 were prepared as described above and then mixed together in a 1:1 ratio prior to administering to mice $(100 \mu \mathrm{L} /$ mouse $)$. A mixture containing both A. muciniphila $\mathrm{YL} 44$ and $B$. vulgatus $\mathrm{RJ} 2 \mathrm{H} 1$ was also prepared for mice in this manner.

\section{Gnotobiotic mice with complex microbiomes}

Germ-free C57BL/6 mice were born and reared in flexible film isolators and maintained under gnotobiotic conditions (temperature $20^{\circ} \mathrm{C}$, relative humidity $60 \%, 14 \mathrm{~h}$ light $/ 10 \mathrm{~h}$ dark cycle) at the University of NebraskaLincoln. All mice were fed an autoclaved chow diet (LabDiet 5K67, Purina Foods, St. Louis, MO) ad libitum. The Institutional Animal Care and Use Committee at the University of Nebraska-Lincoln approved all procedures involving animals (protocols 1215 and 1700).

Four mouse microbiomes MFPL [36], MC608-F-a1 [18, 36], Wild116 $[18,36,37]$, and BALBC.m3 $[36,38]$ were investigated as potential inocula for our experiments. MFPL refers to a C57BL/ $6 \mathrm{~J}$ mouse population of the Max F. Perutz Laboratories in Austria [36]). MC608-F-a1 refers to mice derived from a wild mouse population in the Massif Central region of France previously maintained at the Max Planck Institute for Evolutionary Biology (Plon, Germany; referred to as MC608-F-a1 in [36] and as A in [18]). Wild116 refers to wild mice caught in the United Kingdom [18, 36, 37]. BALBc.m3 refers to a BALB/c mouse population from a laboratory facility in the United Kingdom [36]. To produce standardized inocula of these microbiomes in sufficient amount for all experiments, female germ-free C57BL/6 mice were colonized with cecal contents from the donor mice and maintained under gnotobiotic conditions at the University of NebraskaLincoln for four weeks. Ceca were then collected, stored at $-80^{\circ} \mathrm{C}$, and the contents resuspended $(1: 10 \mathrm{wt} / \mathrm{vol})$ in reduced PBS as previously described [39] at the time of use.

DNA was extracted from the donor inoculum and recipient fecal samples, and microbial composition assessed by paired-end sequencing of the V5-V6 region of the 16S rRNA gene using the MiSeq (Illumina) as previously described [18]. For taxa with a relative abundance greater than $0.03 \%$, Bray-Curtis dissimilarities were calculated using the vegdist function in the $R$ package vegan and then hierarchically clustered using the $R$ function hclust set to the average agglomeration method. Data were then displayed in a heat map using packages ggplot and heatmap 2 (RStudio Team, Boston, MA). Our analysis revealed that the microbiomes of recipient mice clustered with their respective inocula (Fig. S1), suggesting overall successful stable engraftment. The analysis also showed only one of the four microbiomes (MFPL) contained sequences related to A. muciniphila and that the two microbiomes derived from wild mice (MC608-F-a1 and Wild116) lost the family Bacteroidaceae, which contains the genus Bacteroides, upon transplantation into germ-free mice. Cecal contents from the MFPL and MC608-F-a1 mice were pooled and selected as the positive and negative inocula in our study, respectively. The presence and absence of $A$. muciniphila and $B$. vulgatus in both microbiomes were confirmed using species-specific qPCR [38].

\section{Tests of persistence, coexistence, and the importance of} colonization order

To determine to what degree the colonization dynamics of $A$. muciniphila and $B$. vulgatus strains were influenced by the presence or absence of the respective species present in the gut microbiome, female germ-free C57BL/6 mice were colonized with a microbiome identified as either positive (MFPL) or negative (MC608-F-a1) for A. muciniphila and B. vulgatus (week 0) as described above. Two weeks later (week 2), these mice were administered either a mixture of $A$. muciniphila BAA-835 and $B$. vulgatus 8482 or a mixture of $A$. muciniphila YL44 and B. vulgatus $\mathrm{RJ} 2 \mathrm{H} 1$ for a total of two treatments. Each treatment consisted of five mice housed in one cage, and fecal samples were collected weekly at weeks $1,2,3,4,5,6$, and 7 .

To test if colonization could be blocked or influenced by introducing one strain into the gut ecosystem before the other, female germ-free C57BL/6 mice were orally gavaged (week 0 ) with a negative microbiome (devoid of A. muciniphila and B. vulgatus) and a mixture of A. muciniphila BAA-835 and $B$. vulgatus 8482 . Two weeks later (week 2 ), mice were orally gavaged with strains YL44 and RJ2H1. In a separate experiment, the importance of colonization order was tested by inverting the order of strain introduction. Specifically, GF mice were orally gavaged (week 0 ) with a negative microbiome and a mixture of either A. muciniphila YL44 and B. vulgatus $\mathrm{RJ} 2 \mathrm{H} 1$. Two weeks later (week 2), mice were orally gavaged with strains BAA-835 and 8482 . Studies were also performed to test the relative fitness of $A$. muciniphila and $B$. vulgatus strains when introduced at the same time. Female germ-free C57BL/6 mice were colonized with the negative microbiome at week 0 and then administered a mixture of all four strains at week 2. For all studies, each treatment consisted of five mice housed in one cage, and fecal samples were collected weekly at weeks $1,2,3,4,5,6$, and 7. See supplementary information for a description of methods used for $A$. muciniphila subtractive studies in mice. 


\section{Quantitative real-time PCR (qPCR)}

Strain-specific primers were designed to target unique genes not shared among the two test strains (identified using IMG-ER, Joint Genome Institute) [40]. Once unique genes were identified, potential specific primer pairs were generated using Prime3 software [41] and their quality assessed with NetPrimer (Premier Biosoft, San Francisco, CA). Primer specificity was verified bioinformatically by blasting primer sequences against a nonredundant DNA database for bacteria (NCBI) [42] and experimentally by qPCR using DNA isolated from the four strains (B. vulgatus RJ2H1, $B$. vulgatus 8482 , A. muciniphila YL44, and A. muciniphila BAA-835) and the two microbiomes used in our experiments.

Strain-specific primers designed for this study were A. muciniphila BAA835 forward CGGGGACAGTATATCGGGGA, reverse GAGATTCGGATAGC GCACCA; A. muciniphila YL44 forward GCCTTTCTTCAGCAAACGGG, reverse TCACAGCAGTTCAACAGGCA; B. vulgatus 8482 forward TCATCGTGGTC CATTGTCGG, reverse AACACCCCGTCAAAATTGCG; $B$. vulgatus RJ2H1 forward GCCGACGCTTTCTGACAAAA, reverse GAGGCGGCTTTCCATTGTTC. Thermocycling conditions for all four strain-specific primer pairs were: (i) initial denaturation step at $95^{\circ} \mathrm{C}$ for $5 \mathrm{~min}$; (ii) 35 cycles of $95^{\circ} \mathrm{C}$ for $1 \mathrm{~min}$, $64.2^{\circ} \mathrm{C}$ for $30 \mathrm{sec}, 72^{\circ} \mathrm{C}$ for $30 \mathrm{sec}$; and (iii) one $20 \mathrm{~min}$ interval to generate a melting curve by progressively increasing the temperature from $60^{\circ} \mathrm{C}$ to $95^{\circ} \mathrm{C}$.

Species-specific primers for B. vulgatus were also designed using a similar approach as for the strain-specific primers where primers targeted unique genes that were present in this species but absent in all other bacterial sequences reported in NCBI. The species-specific primers targeting B. vulgatus were forward GGCAGCATGGTCTTAGCTTGC, reverse GTGAACATGCGGACTCATGATG. Previously published species-specific primers were used to quantify A. muciniphila [43]. Thermocycling conditions for both $B$. vulgatus and $A$. muciniphila species-specific primer pairs were: (i) initial denaturation step at $95^{\circ} \mathrm{C}$ for $5 \mathrm{~min}$; (ii) 35 cycles of $95^{\circ} \mathrm{C}$ for $1 \mathrm{~min}$, $57^{\circ} \mathrm{C}$ for $45 \mathrm{sec}$, and $72^{\circ} \mathrm{C}$ for $45 \mathrm{sec}$; and (iii) one 20-min interval to generate a melting curve by progressively increasing the temperature from $60^{\circ} \mathrm{C}$ to $95^{\circ} \mathrm{C}$.

All qPCRs were performed using SYBR green (Thermo Scientific, Lithuania) and a Mastercycler Realplex2 (Eppendorf AG, Hamburg, Germany). Specificity was tested using DNA from the strain of interest and the negative microbiomes utilized in this study. Optimal thermocycling conditions for $\mathrm{GPCR}$ were determined via gradient $P C R$ using twelve temperatures between $53^{\circ} \mathrm{C}$ and $63^{\circ} \mathrm{C}$ (equal intervals) [44].

To make qPCR standard curves, aliquots of duplicate log-phase A. muciniphila or B. vulgatus cultures were plated on either BHIs or BHIm media for quantification of colony forming units per milliliter of culture (CFU/mL). A phenol chloroform method [45] was used for DNA extraction. Quantitative PCR including melt curve analysis, was performed on serially diluted (ten-fold) extracted DNA. Bacterial abundance was calculated based on the linear relationship between fluorescence of serially diluted DNA and corresponding CFU/mL [44]. Minimum limit of detection was established to be the lowest DNA dilution at which the relationship between CFU and fluorescence was linear.

\section{Whole metagenomic sequencing (WMS) and bioinformatic analyses}

Metagenomic analysis of complex microbiomes was performed two weeks following transplant into germ-free mice but prior to the introduction of $A$. muciniphila and B. vulgatus test strains. Fecal DNA was extracted as described above [45] and sequenced at 10X depth on the MiSeq (Illumina) by Novogene America (Sacramento, CA). Quality check was performed with FastQC tools [46]. The script bbduk.sh ( $k$ trim $=r, k=23, \operatorname{mink}=11$, hdist $=1$, Joint Genome Institute) was used to remove universal adaptors from samples. C57BL6/J (accession: GCA_000001635.9_GRCm39, NCBI) and Homo sapiens (accession: GCA_000001405.27_GRCh38, NCBI) genomes were utilized to remove mouse and human sequences, respectively, with bbsplit (BBmap tools, Joint Genome Institute). Each cleaned data set was mapped to either $B$. vulgatus 8482 (accession: GCA_000012825.1_ASM1282v1), B. vulgatus RJ2H1 (accession: GCA 002796815.1 ASM279681v1), A. muciniphila BAA-835 (accession: GCA_000020225.1) or A. muciniphila YL44 (accession: GCA_001688765.2) genomes from NCBI using BWA software set to default parameters [47]. SAMtools [47] was utilized to convert files into bam format and extract the mapped sequences. QualiMap [48] was used to determine the genome representation of the four test strains $(8482, \mathrm{RJ} 2 \mathrm{H} 1, \mathrm{BAA}-835$, and $\mathrm{YL} 44)$ in each microbiome using the output "genome fraction coverage" of at least $1 \mathrm{X}$. Results were plotted in Prism 8 (GraphPad Software, San Diego, CA). To determine genome representation of unique genomic sequences between $B$. vulgatus test strains in the positive microbiome, unique core sequences between the strains were extracted using RUCS [49] and verified using Mauve [50]. Mapping to determine and genome representation of strain specific genomic sequences within the positive microbiome was then performed with BWA and QualiMap as described above.

Metagenome de novo sequence assembly was performed with MEGAHIT and evaluated by mapping the original reads to the assembled sequences using BWA software and SAMtools as described above. The software MetaBAT 2 [51] set to default parameters for complex communities was used to collect contigs predicted to the same species into a single bin. Taxonomic identification of bins belonging to $B$. vulgatus and $A$. muciniphila was performed by Blastn with $5.3 \times 10^{6}$ and $2.8 \times 10^{6}$ base pairs for $B$. vulgatus $A$. muciniphila related bins, respectively, which was similar to the average genome sizes reported for these species (NCBI database) $[52,53]$. Genomic representation of test strains against resident $B$. vulgatus and $A$. muciniphila contigs was performed with BWA, SAMtools, and Qualimap as described above. Taxonomic profiles of the metagenomic dataset were generated using MetaPhIAn3 (v3.0.2) [54] with default settings. Taxa presenting in no less than three mice in at least one treatment were retained for data visualization using GraPhIAn (v1.1.3) [55] and the $R$ package pheatmap.

\section{Genomic comparisons between test strains}

IMG/MER tools [40,42] from the Joint Genome Institute were used to calculate genome-wide average nucleotide identity (gANI) and alignment fractions (AF) for A. muciniphila BAA-835 versus YL44 and B. vulgatus 8482 versus $\mathrm{RJ} 2 \mathrm{H} 1$ as previously described [56]. Genome size information from strains reported at NCBI was used to generate histogram distribution using the ggplot2 package in RStudio (RStudio Team, Boston, MA). The IMG/MER phylogenetic profiler tool was used to perform gene context analysis and identify unique genes and protein families (Pfam) for each strain $[40,42,57]$. Classifications of identified protein families were made based on descriptions in the protein family database [58] and the Universal Protein Resource [59]. Pie charts and stacked bar graphs were create using Prism 8 (GraphPad Software, San Diego, CA).

\section{Quantification and statistical analysis}

All longitudinal data were analyzed using two-way ANOVA repeated measures and Tukey test multiple pairwise comparisons using Prism 8 (GraphPad Software). A p-value of 0.05 was considered significant.

\section{RESULTS}

\section{A. muciniphila and $B$. vulgatus strains only colonized gnotobiotic mice harboring complex microbiomes devoid of these species}

To confirm published studies showing that bacterial colonization in the mammalian gut depends on the absence of related bacteria $[17,18,27,60]$, we tested the persistence of $A$. muciniphila and $B$. vulgatus strains in mice harboring complex microbiomes with (positive) and without (negative) these species. We established a microbiome in germ-free mice that contained these species (MFPL) as well as a microbiome that lacked the phylum Verrucomicrobia and the family Bacteroidaceae (MC608-F-a1). After two weeks of colonization, we confirmed the presence/ absence of $A$. muciniphila and $B$. vulgatus by species-specific qPCR. WMS verified the presence of $A$. muciniphila and $B$. vulgatus in the positive microbiome and the absence of these taxa (along with their respective phyla and families) in the negative microbiome (Fig. S2a-C). Approximately $85 \%$ of the genome sequences from test strains A. muciniphila BAA-835 and YL44 and B. vulgatus 8482 and $\mathrm{RJ} 2 \mathrm{H} 1$ were represented in the metagenomes of the positive microbiome (Fig. S2d). In addition, $\sim 70-80 \%$ of the genome sequences from the experimental $A$. muciniphila and $B$. vulgatus strains was shared with the resident $B$. vulgatus and $A$. muciniphila populations present in the positive microbiome (Fig. S2e, f). In contrast, the genomes of all test strains were poorly represented in the negative microbiome (Fig. S2e, f).

We then orally administered experimental strains of $A$. muciniphila and $B$. vulgatus to mice harboring either a positive 
A Positive

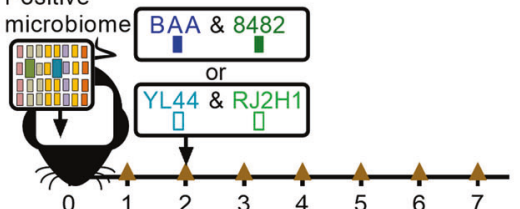

C
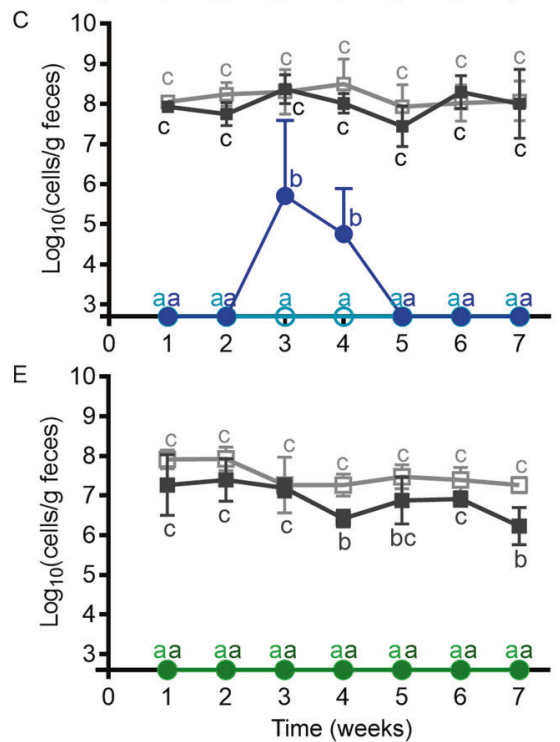

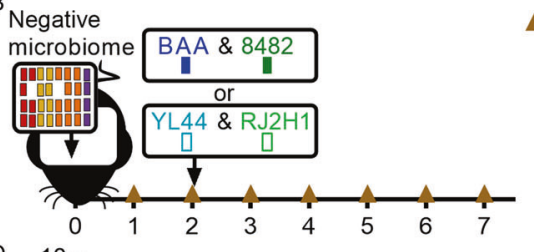

D

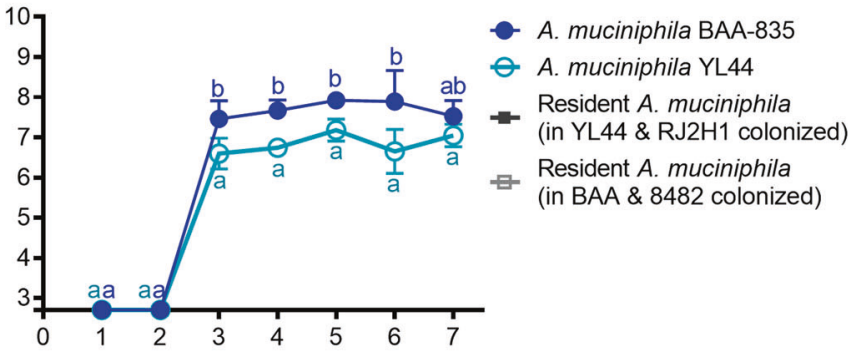

F

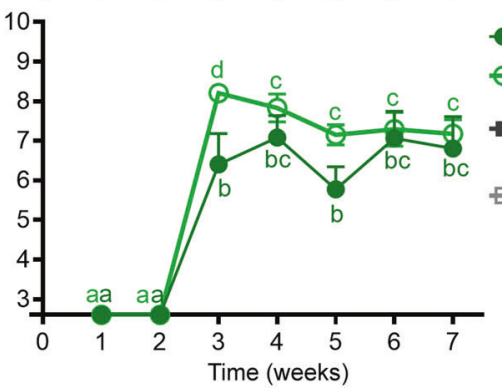

Fecal sample collected

- B. vulgatus 8482

- B. vulgatus RJ2H1

Resident $B$. vulgatus

(in YL44 \& RJ2H1 colonized)

Resident $B$. vulgatus

(in BAA \& 8482 colonized)

Fig. 1 A. muciniphila and B. vulgatus strains only colonized gnotobiotic mice harboring complex microbiomes devoid of these species. Experimental design to test colonization of strains in mice harboring a microbiome with (positive; A) and without (negative; B) A. muciniphila and $B$. vulgatus. Brown triangles represent timepoints for fecal sample collections. Black arrows represent colonization events with microbiomes or $A$. muciniphila and B. vulgatus strains. Week 2 fecal samples were collected prior to inoculating with test strains. Abundance of A. muciniphila species (gray), strain BAA-835 (dark blue), and strain YL44 (light blue) in mice harboring either a positive (C) or a negative (D) microbiome. Abundance of B. vulgatus species (gray), strain 8482 (dark green), and strain RJ2H1 (light green) in mice harboring either a positive (E) or a negative (F) microbiome. Values are presented as mean \pm the standard deviation. Time points with different letters are significantly $(p<0.05)$ different from one another at indicated timepoints by two-way ANOVA repeated measures and Tukey test multiple pairwise comparisons in each plot.

or negative microbiome. Using strain-specific qPCR (Fig. 1A), we found that neither the $A$. muciniphila nor the $B$. vulgatus test strains colonized mice carrying a microbiome that contained these species (Fig. 1C, D). In contrast, stable persistence of test strains was achieved in mice harboring the negative microbiome that was naturally devoid of $A$. muciniphila and B. vulgatus (Fig. 1D, F). These results suggest that colonization of exogenous bacterial strains can occur in the absence, but not presence, of the same species (or species representing the genetic potential of the incoming strains) in the resident microbiome, thus confirming previous findings in both mice and humans $[17,18]$.

\section{Colonization of $A$. muciniphila, but not $B$. vulgatus, was strictly governed by competitive exclusion, while priority effects were detectable for both species}

Although the findings above suggest that concepts such as competitive exclusion or limiting similarity pertain to gut ecosystems [61], they do not provide direct evidence because the differences between the positive and negative microbiomes pertained not only to the target species but also to their respective phyla and families. Our next experiments therefore aimed to specifically evaluate the outcome of strain-to-strain competition and test whether it resulted in competitive exclusion. We first colonized germ-free mice with a "permissive" negative microbiome devoid of $A$. muciniphila and $B$. vulgatus and a mixture of both A. muciniphila BAA-835 and B. vulgatus 8482 . Two weeks later, mice were colonized with a mixture of both $A$. muciniphila YL44 and B. vulgatus RJ2H1 (Fig. 2A). A second cohort of mice harboring permissive microbiomes were colonized with both
A. muciniphila YL44 and B. vulgatus $\mathrm{RJ} 2 \mathrm{H} 1$ first, followed by A. muciniphila BAA-835 and B. vulgatus 8482 two weeks later (Fig. 2B). Finally, a third cohort of mice colonized with the permissive microbiome was used to test all four strains together to determine fitness differences of the strains (Fig. 2C).

For A. muciniphila, we observed that the strain arriving first stably colonized, while the second was only temporarily detected, indicating competitive exclusion (Fig. 2D, E). Notably, competition outcomes were strictly dependent on arrival order, with the first colonizer excluding the later colonizer, thus demonstrating that priority effects are of paramount importance. Priority effects were strong enough to abrogate the fitness differences observed between the two $A$. muciniphila strains. Strain BAA-835 outcompeted YL44 when it colonized first, although it was outcompeted by YL44 when both strains were inoculated together (Fig. 2F).

In contrast to the findings obtained for $A$. muciniphila, both strains of $B$. vulgatus were able to stably colonize independently of strain arrival succession (Fig. 2G-I). Despite stable coexistence, the two strains still influenced one another's abundance, indicating competitive interactions that were further influenced by priority effects. Although the sum total abundance of the two B. vulgatus strains was equivalent across all colonization scenarios (Fig. S3), the maximum abundance levels for individual strains were significantly higher (mean estimate of difference $0.5-1.0 \log , p<$ $0.05)$ when they colonized first as compared to when colonizing second (Fig. $2 \mathrm{G}$ vs. H). Second, the higher population level of strain $\mathrm{RJ} 2 \mathrm{H} 1$ ( $\sim 10^{8}$ cells $/ \mathrm{g}$ feces) compared to $8482\left(\sim 10^{6}\right.$ cells $/ \mathrm{g}$ feces) observed when $\mathrm{RJ} 2 \mathrm{H} 1$ colonized first or at the same time as 8482 

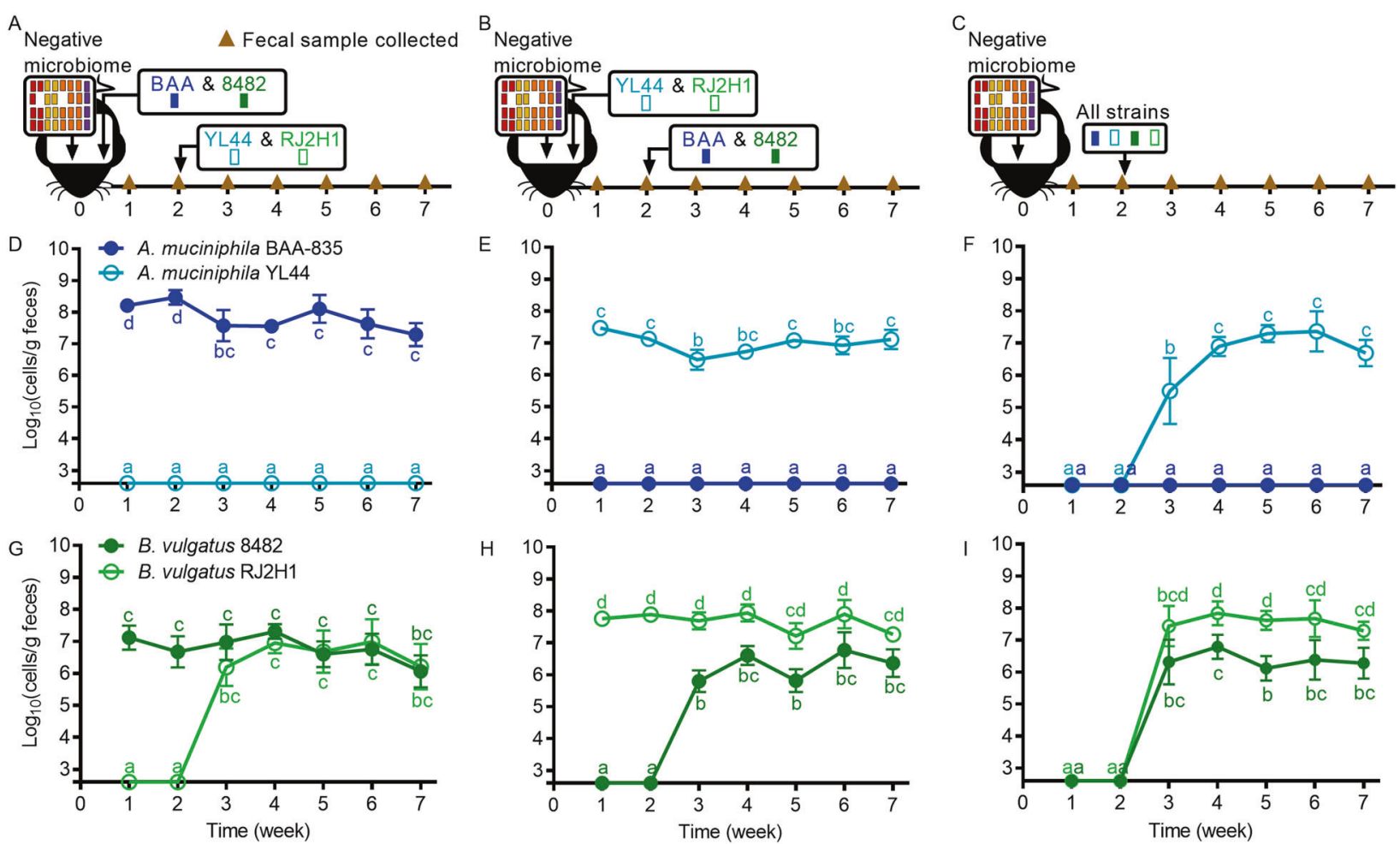

Fig. 2 Colonization of $A$. muciniphila, but not $B$. vulgatus, was strictly governed by competitive exclusion, while priority effects were detectable for both species. All mice were colonized at week 0 with a negative microbiome devoid of $A$. muciniphila and $B$. vulgatus. Competition experiments with strains of $A$. muciniphila and $B$. vulgatus were performed as follows: (A, D, G) $A$. muciniphila BAA-835 and $B$. vulgatus 8482 were introduced at week 0 prior to introduction of $A$. muciniphila YL44 and B. vulgatus RJ2H1 at week 2. B, E, H A. muciniphila YL44 and B. vulgatus $\mathrm{RJ} 2 \mathrm{H} 1$ were introduced at week 0 prior to introduction of $A$. muciniphila BAA-835 and B. vulgatus 8482 at week 2 . C, F, I $A$. muciniphila and $B$. vulgatus strains were all introduced into mice at the same time. D-F Abundance of $A$. muciniphila strain BAA-835 (dark blue) and strain YL44 (light blue) in feces. G-I Abundance of B. vulgatus strain 8482 (dark green) and strain RJ2H1 (light green) in feces. Week 2 fecal samples were collected prior to inoculating with test strains. Values are presented as mean \pm the standard deviation. Time points with different letters are significantly $(p<0.05)$ different from one another by two-way ANOVA repeated measures and Tukey test multiple pairwise comparisons among treatments of $A$. muciniphila (D-F) or B. vulgatus (G-I).

was no longer detectable when strain 8482 was introduced first (both strains at $\sim 10^{7}$ cells $/ g$ feces). These findings indicate that although the two $B$. vulgatus strains coexisted and were not subjected to strict competitive exclusion, the two strains still affected each other's population levels and priority effects clearly influenced competition outcomes.

\section{Strain-to-strain differences in traits enabling niche differentiation may explain the distinct competition outcomes between $A$. muciniphila and $B$. vulgatus strains}

Similarities between bacteria increase competition for resources and may result in competitive exclusion [62]. However, differences in resource requirements can lead to resource partitioning and niche differentiation and thus constitute a stabilizing mechanism that increases the chance of coexistence [25]. We therefore sought to investigate whether genetic relationships could explain the differences in coexistence patterns observed between $A$. muciniphila (strict competitive exclusion) and $B$. vulgatus (coexistence with competitive interactions) strains. An assessment of genetic relatedness using genome-wide average nucleotide identity (gANI) and alignment fraction (AF) metrics [56] revealed that the genomes of the two A. muciniphila strains were more similar to one another than those of the $B$. vulgatus strains (gANI values of $99.10 \mathrm{vs.} 98.68$ and $\mathrm{AF}$ values of 93.37 vs. 75.58 for $A$. muciniphila and B. vulgatus, respectively; Table 1 ). We also observed that the genomes of A. muciniphila strains were smaller than those of $B$. vulgatus strains (Fig. 3A) and consistent in size with organisms exhibiting specialist behaviors [63], such as the mucin degradation/utilization for which
A. muciniphila is highly specialized [64]. Each A. muciniphila strain differed from one another in only a few unique encoded proteins related to processes of gene regulation and nitrogen metabolism (Fig. 3B, D). In contrast, the $B$. vulgatus strains differed by multiple proteins related to processes of gene regulation, carbohydrate binding and metabolism, phage infection, stress responses, and protein degradation (Fig. 3C, E).

Consistent with these observations, B. vulgatus strains 8482 and $\mathrm{RJ} 2 \mathrm{H} 1$ differed in terms of the carbohydrate substrates they utilized in vitro (Table S1). Specifically, strain 8482 exhibited superior growth on $\mathrm{N}$-acetyl glucosamine and chondroitin sulfate as well as on simple sugars such as fructose and glucose, whereas strain $\mathrm{RJ} 2 \mathrm{H} 1$ grew well on several starches, including amylopectin, glycogen, pullulan, and rhamnogalacturonan I, and xylan.

Altogether, these findings suggest that competitive exclusion between $A$. muciniphila strains is likely related to high genetic similarity. In contrast, the coexistence of $B$. vulgatus strains may be determined by unique genes and patterns of carbohydrate metabolism whereby strain 8482 specializes towards hostderived carbohydrates and simple dietary sugars while strain $\mathrm{RJ} 2 \mathrm{H} 1$ specializes on complex dietary substrates to promote niche differentiation. Importantly, $\sim 70 \%$ of the genome sequences found to be unique to either B. vulgatus 8482 or $\mathrm{RJ} 2 \mathrm{H} 1$ in direct genome comparisons between these strains, which may encode the traits that enable niche partitioning, was represented in the positive microbiome (Fig. 3f), thus providing an explanation for why this microbiome excluded both B. vulgatus strains (Fig. 1E). 
Table 1. Genome-wide average nucleotide identities among the strains utilized in this study.

\begin{tabular}{lllccc|} 
Genome1 Name & Genome2 Name & ANI1-> 2 & ANI2->1 & AF1-> 2 & AF2->1 \\
\hline Bacteroides vulgatus ATCC 8482 & Bacteroides vulgatus RJ2H1 & 98.7 & 98.7 & 75.6 & 75.6 \\
\hline Bacteroides vulgatus ATCC 8482 & Akkermansia muciniphila YL44 & 65.5 & 65.7 & 1.0 \\
\hline Akkermansia muciniphila ATCC BAA-835 & Akkermansia muciniphila YL44 & 99.1 & 99.1 & 93.4 \\
\hline Akkermansia muciniphila ATCC BAA-835 & Bacteroides vulgatus RJ2H1 & 65.7 & 65.7 & 90.7 \\
\hline
\end{tabular}

Average nucleotide identity (ANI) and Alignment Fraction (AF) are measuring genetic relatedness. ANI1 and AF1 are calculated with Genome 1 as the reference. ANI2 and AF2 are calculated with Genome 2 as the reference.

\section{A subtractive antibiotic strategy enabled replacement of an established $A$. muciniphila strain}

Our observations for A. muciniphila suggest that the establishment of a new strain of this species is prevented via competitive exclusion by a resident strain of the same species whose fitness is enhanced through priority effects. This finding provides a mechanism underlying the difficulty in establishing new strains within the gut microbiota and implies that successful colonization of novel $A$. muciniphila strains depends upon the reduction or removal of a pre-existing, related strain within the microbiome using a subtractive approach [15]. One potential strategy for such modulation is antibiotic treatment [65]. We therefore hypothesized that an antibiotic regimen would allow us to replace an established strain of $A$. muciniphila with a novel strain. We selected candidate antibiotics based on Derrien et al., who reported that A. muciniphila was susceptible to ampicillin (AMP) [64] and other reports describing antibiotics with effects on $A$. muciniphila or Verrucomicrobia [31, 66, 67]. Candidate antibiotics were first screened in vitro for their ability to attenuate growth of A. muciniphila BAA-835 and YL44 (Table S2). Macrolide tylosin tartrate $(M T T)$, clarithromycin (CLA), and AMP all limited the growth of BAA and YL44 in vitro.

To systematically test the efficacy of an antibiotic-based subtractive strategy, germ-free mice were first conventionalized with a permissive microbiota devoid of $A$. muciniphila and then colonized one week later with strain BAA-835. One week after the addition of BAA-835, mice received either CLA, MTT, AMP, AMP + $M T T, A M P+M T T+C L A$, or no antibiotics in their drinking water for five days. On the fifth day, mice receiving antibiotics were returned to regular drinking water and began receiving daily gavages of YL44 for five days (Fig. 4A). In agreement with findings from our previous experiment (Fig. 2d), YL44 could not colonize control mice and did not influence BAA-835 population levels (Fig. 4B). Treatment with either MTT or CLA resulted in the same outcomes as observed for control mice (Fig. 4C and D). In contrast, administering AMP alone or in combination with MTT and/or CLA depleted the existing BAA strain to undetectable levels and enabled stable colonization of YL44 for the duration of the fiveweek experiment (Fig. 4E-G). Together, these results demonstrate that subtractive antibiotic treatment can be used as a strategy to successfully remove a pre-existing $A$. muciniphila strain from an assembled gut microbial community and replace it with a novel strain that would otherwise be excluded by competitive exclusion. These experiments also further confirm that the competitive interactions between $A$. muciniphila strains detected in previous experiments are not due to interactions with $B$. vulgatus.

\section{DISCUSSION}

Recent studies suggest that colonization of an incoming microbe in a microbiome is determined by the presence of closely related inhabitants $[17,18,27,60]$, thus suggesting that principles such as competitive exclusion apply to gut ecosystems. However, the applicability of these concepts has not been empirically established, nor have there been attempts to apply them to exchange strains in an assembled community. Using systematic experiments in gnotobiotic mice, we found that $A$. muciniphila strains, which have narrow and likely overlapping niches, excluded one another from the microbiome whereas $B$. vulgatus strains could coexist, likely through niche partitioning, but still showed ecological interactions pointing to competition. We observed that competitive interactions between both A. muciniphila and B. vulgatus strains were influenced by time of arrival, thus establishing the importance of priority effects as a determinant of coexistence. Finally, we demonstrated that antibiotic treatments can be used to replace $A$. muciniphila strains within an assembled microbiome. Altogether, our results suggest that important aspects of coexistence theory (e.g., the ability to partition niches and the impact of priority effects on fitness differences) determine strain competition outcomes in gut ecosystems and suggest that such principles can be applied to design strategies that modulate microbiomes.

According to modern coexistence theory, coexistence in an ecosystem is determined by the degree to which members differ in fitness (i.e., equalizing mechanisms), their niches (i.e., stabilizing mechanisms), and their time of arrival $[25,26,68,69]$. Our findings agree with these principles, which can provide an explanation for the profound differences between $A$. muciniphila and $B$. vulgatus with respect to coexistence. $A$. muciniphila colonizes the mucus layer [29], an anatomically-defined structure composed primarily of mucin agglomerates [70], and preferentially metabolizes mucin over other carbohydrates $[64,71]$, thus restricting the variability of resources in its ecological niche. These behaviors are also consistent with the small genomic differences between strains BAA-835 and YL44, indicating that these strains are ecologically very similar. Consequently, one would predict that stabilizing mechanisms are reduced between competing $A$. muciniphila strains because they are unable to partition niches, which should result in strict competitive exclusion where the better competitor excludes the other strain. These assumptions are supported by our current observations for $A$. muciniphila. However, our findings indicate that the ability of these strains to compete (i.e., fitness) is influenced by arrival order. Specifically, the less fit $A$. muciniphila strain won the competition when it arrived first. Our results therefore confirm the importance of priority effects in gut ecosystems $[18,26]$ and demonstrate that these effects can be strong enough to overcome inherent fitness differences between A. muciniphila strains.

We were surprised to not see a competitive advantage for the $A$. muciniphila strain isolated from mice (YL44) over the strain of human origin (BAA-835) given that host adaptation has been shown for other bacteria closely associated with host epithelia (e.g., Limosilactobacillus reuteri) [72, 73]. It therefore appears that host origin plays no role in influencing the competitive abilities of A. muciniphila. However, one should consider that the microbiomes of mice housed in modern research vivaria are often highly artificial [74], and the microbiome derived from wild mice in our study did not contain $A$. muciniphila. We therefore screened the literature to determine if $A$. muciniphila is truly of murine origin. Results from several studies confirmed our observation that A. muciniphila has not been detected in the microbiomes of wild mice $[75,76]$. Overall, these findings suggest that $A$. muciniphila 
A
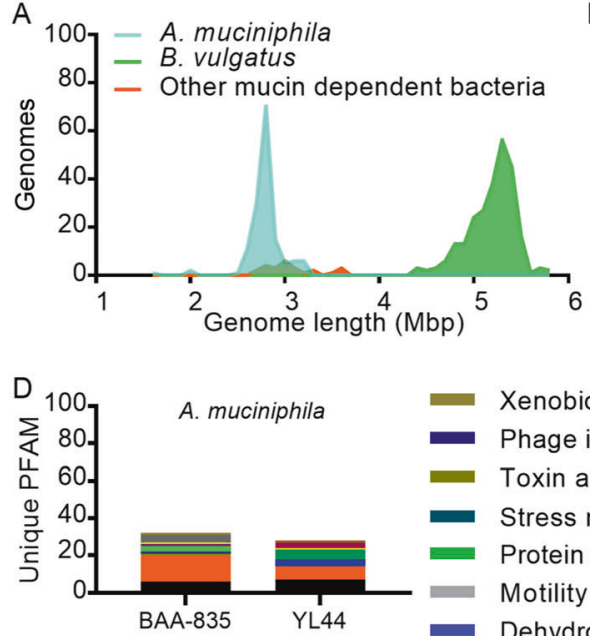

E
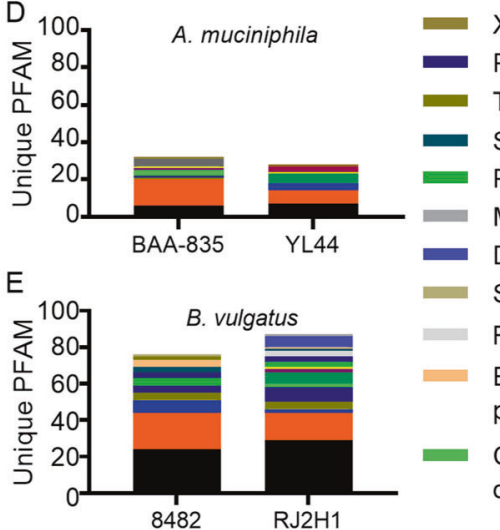

B

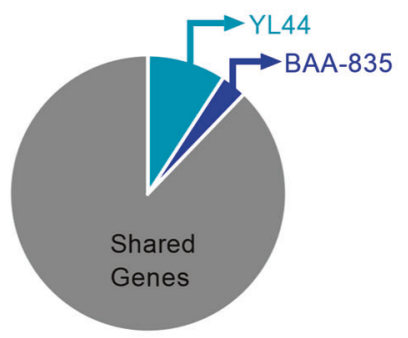

Xenobiotic metabolism

Phage infection

Toxin antitoxin system

Stress response

Protein degradation

Motility

Dehydrogenation

Sulfur metabolism

Ribosome structure

Eukariotic related process

Cell wall synthesis and degradation

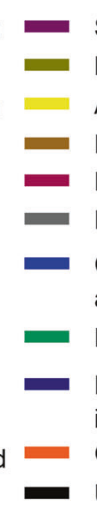

Secretion system

Membrane protein ATP exchange

Peroxidation

Protein synthesis

Nitrogen metabolism

Carbohydrate synthesis and metabolism

Defense system

Protein activity and interaction

Gene expression

Unknown functions
C

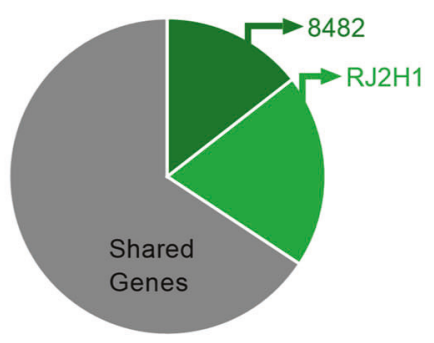

F Strain specific genomic content

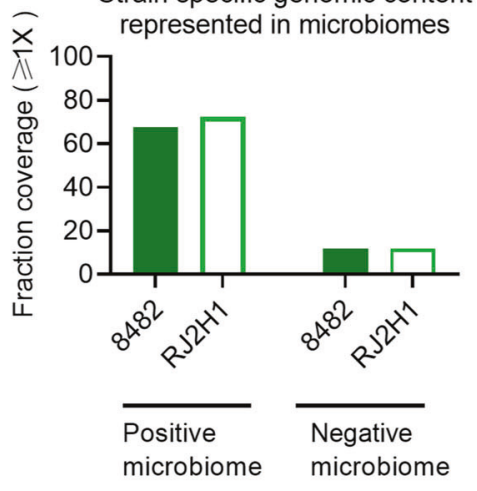

Fig. 3 Genomic differences between A. muciniphila and B. vulgatus strains. A Distribution of bacterial genome sizes. Mbp $=$ Millions of base pairs of total DNA sequence length for each strain in the NCBI genome database. B Shared (gray) and unique genes for A. muciniphila BAA-835 (dark blue) and YL44 (light blue) in direct genome comparisons. C Shared (gray) and unique genes for B. vulgatus 8482 (dark green) and RJ2H1 (light green). Sizes of pie charts are scaled to represent proportional differences between $A$. muciniphila and $B$. vulgatus genomes. Biological processes related to unique protein families are depicted for $A$. muciniphila (D) and B. vulgatus (E) strains. Each protein is grouped by colors that represent an individual biological process. F Fraction of the genome sequences unique to $B$. vulgatus 8482 or RJ2H1 (in genomic comparisons between the two strains) that is represented in the positive and negative (permissive) pretreatment microbiomes.

YL44 shares no evolutionary relationship with mice, thus explaining why it does not exhibit a stronger ecological performance when compared with a human isolate.

Unlike A. muciniphila strains, $B$. vulgatus strains do not exclude each other. This observation can be explained by the existence of stabilizing mechanisms that lead to niche differentiation. In contrast to $A$. muciniphila, whose niche is restricted to the mucus layer, $B$. vulgatus colonizes food particles and scattered luminal regions adjacent to the colonic mucosa $[77,78]$. The $B$. vulgatus niche is therefore likely much more complex and dynamic than that of $A$. muciniphila and provides a larger range of substrates that become accessible by different strains. Indeed, the larger genomes for $B$. vulgatus compared to $A$. muciniphila suggest that these strains are generalists [14]. Consistently, $B$. vulgatus strains 8482 and $\mathrm{RJ} 2 \mathrm{H} 1$ also differed in diversity of carbohydrate and protein degradation capabilities and substrate binding/utilization, which suggests that they are able to partition niches and stably coexist even if equalizing mechanisms are low due to fitness differences [25]. Despite the stable coexistence of $B$. vulgatus isolates, we still detected competitive interactions that were influenced by priority effects. $B$. vulgatus strains achieved a higher colonization level when they arrived first, and fitness differences could be overcome through early arrival. Overall, our observations for $B$. vulgatus strains are consistent with coexistence theory in that strains capable of partitioning niches can coexist. However, our experiments have not identified niche differentiation within $B$. vulgatus on a mechanistic level, nor did they assess competitive interactions among closely related species (as mice harboring the negative microbiome lacked Bacteroidaceae) or how such interactions affect the niche of $B$. vulgatus. Future studies to experimentally determine the mechanisms of niche partitioning within $B$. vulgatus in the context of complex communities are therefore required. Regardless of the exact mechanisms, a novel and important finding for our understanding of the gut ecosystem is that even if strains stably coexist, their population levels are still affected by competition and influenced by priority effects.

Taken together, our findings suggest that key aspects of modern coexistence theory can be applied to understand fundamental characteristics of gut microbial communities. In accordance with that theory, species and strains with similar fitness levels can coexist when niche differences are sufficiently large to reduce overlap in resource usage [25]. In contrast, if niches overlap too much, then fitness differences prevent coexistence and result in competitive exclusion [25]. Our observations for A. muciniphila provide experimental evidence to support previous findings that point to competitive exclusion as a mechanism for colonization resistance $[17,18,27,60]$. Most importantly, our findings agree with observations for strain coexistence in humans $[75,79,80]$. In particular, Truong et al. showed that most individuals harbor only one strain of $A$. muciniphila but tend to carry multiple resident $B$. vulgatus strains [80], suggesting that the ecological principles underpinning strain coexistence in mice may also apply to humans.

Of particular significance is our demonstration that priority effects can be strong enough to modify and even reverse fitness differences between strains and consequently alter competition outcomes, regardless of whether they result in strict competitive exclusion or coexistence. By demonstrating that arrival order alters fitness differences among strains and ultimately reverses the outcome of their competition, our findings extend previous research [18] describing that priority effects can influence the persistence of individual colonizers and the historical contingency of microbiome assembly. Priority effects, in the context of modern coexistence theory, can therefore provide mechanistic explanations for key characteristics of gut ecosystems. First, competitive exclusion, in combination with priority effects, 
A

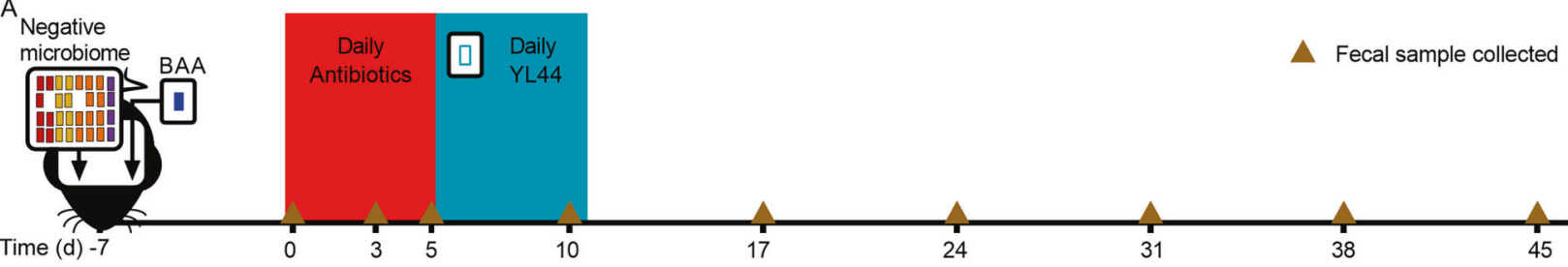

$B \quad$ Control (No Antibiotics)

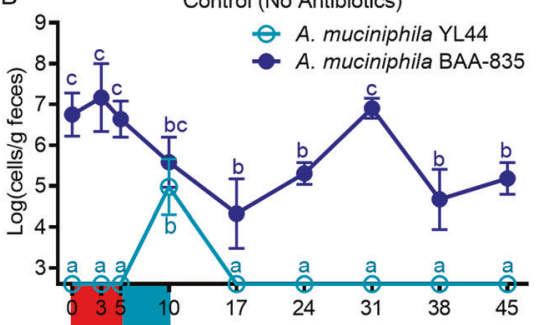

E

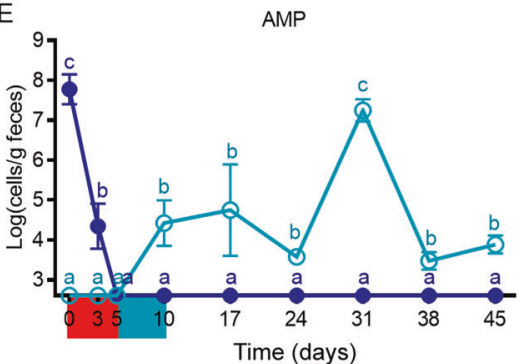

C

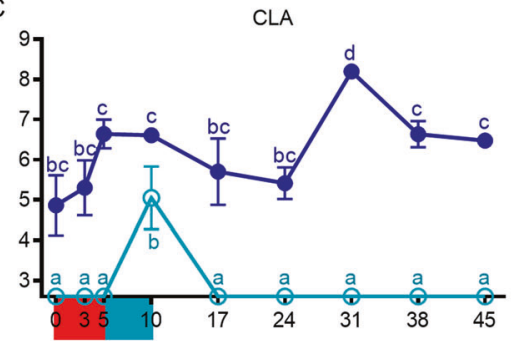

F

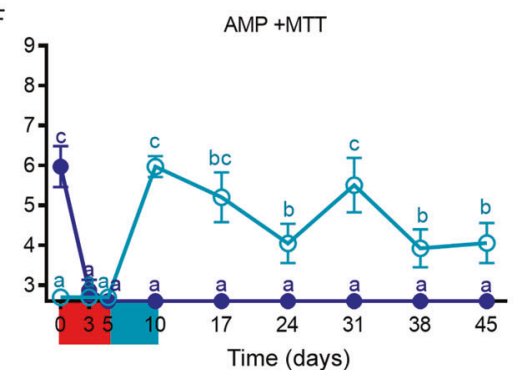

D

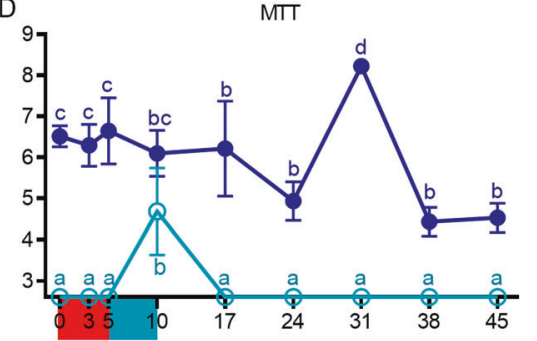

G

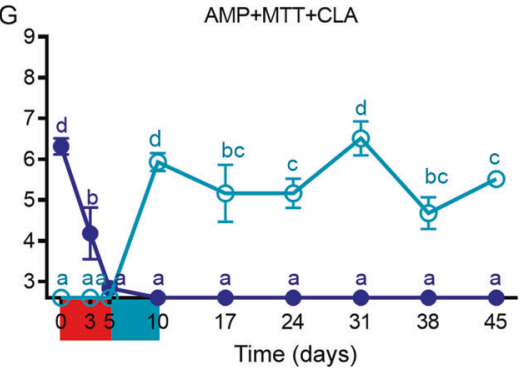

Fig. 4 A subtractive antibiotic strategy enabled replacement of an established $\boldsymbol{A}$. muciniphila strain. A Experimental design depicting conventionalization of germ-free mice with a negative microbiota devoid of $A$. muciniphila and colonization with strain BAA-835 at day - 7. On day 0 , mice were treated with antibiotics or regular drinking water for five days. On day 5 , mice receiving antibiotics were returned to regular drinking water and fecal samples were collected. Also on day 5, mice began receiving daily gavages of YL44 for five days. Brown triangles represent timepoints for fecal sample collections. Abundance of $A$. muciniphila BAA-835 (dark blue) and $A$. muciniphila YL44 (light blue) in feces of mice not treated with antibiotics $(\mathbf{B})$ or treated with CLA alone (C), D MTT alone, E AMP alone, F AMP + MTT, G AMP + MTT + CLA. Values

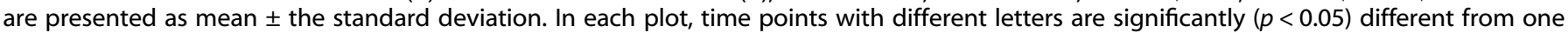
another by two-way ANOVA repeated measures and Tukey test multiple pairwise comparisons.

provides a mechanism that enhances colonization resistance of gut microbiomes as it endows the established organism with a fitness advantage over later arrivals, thereby providing an explanation for microbiome stability, resistance, and resilience $[81,82]$. Second, priority effects could explain why maternalderived bacteria, which are likely to arrive early, are more stable colonizers compared to non-maternal strains [83, 84]. Third, priority effects can influence the abundance of coexisting community members, thus offering a mechanism by which arrival order (which is likely to be largely stochastic) creates differences in gut microbiota composition and explains, in part, the substantial interindividual variation observed in gut microbiomes [18]. We acknowledge that we have not established the mechanisms by which priority effects emerge. Such effects may manifest through ecological (e.g., niche pre-emption) or evolutionary mechanisms (e.g., by early colonizers adapting to the host, thereby becoming more competitive), but could also be influenced by the host immune system if early colonizing strains induce immune responses that benefit their persistence. Future studies should focus on elucidating these mechanisms.

Considering these ecological concepts, there are substantial practical implications for successfully introducing a new strain into the gut microbiota: (i) closely-related established strains must be absent [17,38]; (ii) the incoming strain, which is disadvantaged by priority effects, must outcompete the resident strain; or (iii) the resident strain currently occupying the niche must first be removed through subtractive approaches. The third strategy has been both proposed and applied in some fecal microbiota transplant (FMT) studies [17, 85, 86], and a recent meta-analysis of newly generated and available metagenomes from post-FMT patient samples revealed that antibiotic pretreatment enhanced donor strain engraftment [19]. Our findings provide a mechanistic foundation for the effect of antibiotics on strain engraftment by demonstrating that a subtractive approach based on antibiotic treatment could indeed be used to replace an established A. muciniphila strain with a new one. Specifically, administration of antibiotics suppressed the abundance of the competing, earlyarriving $A$. muciniphila strain and opened a niche for the latearriving strain to colonize. Subtractive approaches such as antibiotics may therefore enable microbiome modulation by both suppressing competing strains and opening niches for new colonizers. We acknowledge that the use of broad-spectrum antibiotics such as ampicillin to replace strains has substantial disadvantages for translation into humans. More targeted subtractive methods, including the use of bacteriophages [87] or CRISPR/cas systems [88], are likely more desirable, and, if applied in agreement with ecological theory, could pave the way for precision tools to modulate microbiomes. Importantly, our study provides the proof-of-concept that such approaches can be successful.

In conclusion, this study demonstrates the applicability of the central aspects of modern coexistence theory to gut ecosystems and that such theory can be used to understand engraftment of incoming microbes. Our findings provide potential explanations for many fundamental characteristics of the gut microbiome, including stability, colonization resistance, enhanced stability of maternally-acquired strains, and drivers of inter-subject variation. Furthermore, this work informs future intervention studies aimed 
at modulating gut ecosystems using live microbes, which will likely need to be personalized based on an individual's baseline microbiome and attempt to remove competitors through subtractive approaches. Admittedly, our study only tested two strains each of two important bacterial species, and the ecological principles governing other microbiota members and the context in which they apply, might differ. Future research should extend our work to include additional strains with known niche characteristics, evaluate their competition in the context of the wider microbial community, and focus efforts on the exact mechanisms by which strains coexist or compete and priority effects emerge. Additionally, gnotobiotic mouse models could be designed to facilitate the investigation of interspecies interactions at various taxonomic levels (e.g., including other Bacteroides species with well-characterized niches [35]). Studies could also be performed that combine gnotobiotic models of disease with genetic mutants to determine the bacterial traits that contribute to niche partitioning to further understand the exact mechanisms by which microbes coexist in gut ecosystems and how their relationships are influenced by pathologies that alter the gut microbiota.

\section{DATA AVAILABILITY}

The datasets generated during the current study are available in Mendeley with the identifier https://doi.org/10.17632/gbc76stz42.2. The metagenomic data that support the findings of this study have been deposited in the NCBI Sequence Read Archive under the BioProject ID PRJNA788901.

\section{REFERENCES}

1. Brugiroux S, Beutler M, Pfann C, Garzetti D, Ruscheweyh HJ, Ring D, et al. Genomeguided design of a defined mouse microbiota that confers colonization resistance against Salmonella enterica serovar Typhimurium. Nat Microbiol. 2016;2:16215.

2. Buffie CG, Bucci V, Stein RR, McKenney PT, Ling L, Gobourne A, et al. Precision microbiome reconstitution restores bile acid mediated resistance to Clostridium difficile. Nature 2015;517:205-8.

3. He M, Shi B. Gut microbiota as a potential target of metabolic syndrome: the role of probiotics and prebiotics. Cell Biosci. 2017;7:54.

4. Ma W, Mao Q, Xia W, Dong G, Yu C, Jiang F. Gut microbiota shapes the efficiency of cancer therapy. Front Microbiol. 2019;10:1050.

5. Rodriguez J, Hiel S, Neyrinck AM, Le Roy T, Potgens SA, Leyrolle Q, et al. Discovery of the gut microbial signature driving the efficacy of prebiotic intervention in obese patients. Gut 2020;69:1975-87.

6. Schubert AM, Sinani H, Schloss PD. Antibiotic-induced alterations of the murine gut microbiota and subsequent effects on colonization resistance against Clostridium difficile. mBio 2015;6:e00974.

7. Vivarelli S, Salemi R, Candido S, Falzone L, Santagati M, Stefani S, et al. Gut microbiota and cancer: From pathogenesis to therapy. Cancers (Basel). 2019;11:38.

8. Pasolli E, Truong DT, Malik F, Waldron L, Segata N. Machine learning metaanalysis of large metagenomic datasets: Tools and biological insights. PLoS Comput Biol. 2016;12:e1004977.

9. Walters WA, Xu Z, Knight R. Meta-analyses of human gut microbes associated with obesity and IBD. FEBS Lett. 2014;588:4223-33.

10. Rastelli M, Knauf C, Cani PD. Gut microbes and health: A focus on the mechanisms linking microbes, obesity, and related disorders. Obes (Silver Spring) 2018;26:792-800.

11. Sonnenburg JL, Backhed F. Diet-microbiota interactions as moderators of human metabolism. Nature 2016;535:56-64.

12. Costello EK, Stagaman K, Dethlefsen L, Bohannan BJ, Relman DA. The application of ecological theory toward an understanding of the human microbiome. Science 2012;336:1255-62.

13. Koskella B, Hall $\sqcup$, Metcalf CJE. The microbiome beyond the horizon of ecological and evolutionary theory. Nat Ecol Evol. 2017;1:1606-15.

14. Walter J, Ley R. The human gut microbiome: Ecology and recent evolutionary changes. Annu Rev Microbiol. 2011;65:411-29.

15. Walter J, Maldonado-Gomez MX, Martinez I. To engraft or not to engraft: An ecological framework for gut microbiome modulation with live microbes. Curr Opin Biotechnol. 2018;49:129-39.

16. Le Roy T, Debedat J, Marquet F, Da-Cunha C, Ichou F, Guerre-Millo M, et al. Comparative evaluation of microbiota engraftment following fecal microbiota transfer in mice models: Age, kinetic and microbial status matter. Front Microbiol. 2018;9:3289.

17. Maldonado-Gomez MX, Martinez I, Bottacini F, O'Callaghan A, Ventura $M$, van Sinderen D, et al. Stable engraftment of Bifidobacterium longum AH1206 in the human gut depends on individualized features of the resident microbiome. Cell Host Microbe. 2016;20:515-26.

18. Martinez I, Maldonado-Gomez MX, Gomes-Neto JC, Kittana H, Ding H, Schmaltz R, et al. Experimental evaluation of the importance of colonization history in earlylife gut microbiota assembly. Elife. 2018;7:e36521.

19. Podlesny D, Durdevic M, Paramsothy S, Kaakoush NO, Högenauer C, Gorkiewicz $\mathrm{G}$, et al. Intraspecies strain exclusion, antibiotic pretreatment, and donor selection control microbiota engraftment after fecal transplantation. medRxiv. 2021;08.18.21262200.

20. Li SS, Zhu A, Benes V, Costea Pl, Hercog R, Hildebrand F, et al. Durable coexistence of donor and recipient strains after fecal microbiota transplantation. Science 2016;352:586-89.

21. Seekatz AM, Aas J, Gessert CE, Rubin TA, Saman DM, Bakken JS, et al. Recovery of the gut microbiome following fecal microbiota transplantation. mBio 2014;5: e00893-00814.

22. Shahinas D, Silverman M, Sittler T, Chiu C, Kim P, Allen-Vercoe E, et al. Toward an understanding of changes in diversity associated with fecal microbiome transplantation based on 16S rRNA gene deep sequencing. mBio. 2012;3:e00338-12.

23. Hardin G. The competitive exclusion principle. Science 1960;131:1292-7.

24. Stecher B, Chaffron S, Kappeli R, Hapfelmeier S, Freedrich S, Weber TC, et al. Like will to like: Abundances of closely related species can predict susceptibility to intestinal colonization by pathogenic and commensal bacteria. PLoS Pathog. 2010;6:e1000711.

25. Chesson P. Mechanisms of maintenance of species diversity. Annu Rev Ecol Syst. 2000;31:343-66.

26. Grainger TN, Letten AD, Gilbert B, Fukami T. Applying modern coexistence theory to priority effects. Proc Natl Acad Sci USA. 2019;116:6205-10.

27. Lee SM, Donaldson GP, Mikulski Z, Boyajian S, Ley K, Mazmanian SK. Bacterial colonization factors control specificity and stability of the gut microbiota. Nature 2013;501:426-9.

28. Onderdonk A, Marshall B, Cisneros R, Levy SB. Competition between congenic Escherichia coli K-12 strains in vivo. Infect Immun. 1981;32:74-9.

29. Everard A, Belzer C, Geurts L, Ouwerkerk JP, Druart C, Bindels LB, et al. Cross-talk between Akkermansia muciniphila and intestinal epithelium controls dietinduced obesity. Proc Natl Acad Sci USA. 2013;110:9066-71.

30. Shin NR, Lee JC, Lee HY, Kim MS, Whon TW, Lee MS, et al. An increase in the Akkermansia spp. population induced by metformin treatment improves glucose homeostasis in diet-induced obese mice. Gut 2014;63:727-35.

31. Dingemanse C, Belzer C, van Hijum SA, Gunthel M, Salvatori D, den Dunnen JT, et al. Akkermansia muciniphila and Helicobacter typhlonius modulate intestinal tumor development in mice. Carcinogenesis 2015;36:1388-96.

32. Png CW, Linden SK, Gilshenan KS, Zoetendal EG, McSweeney CS, Sly LI, et al. Mucolytic bacteria with increased prevalence in IBD mucosa augment in vitro utilization of mucin by other bacteria. Am J Gastroenterol. 2010;105:2420-8.

33. Zhai R, Xue X, Zhang L, Yang X, Zhao L, Zhang C. Strain-specific anti-inflammatory properties of two Akkermansia muciniphila strains on chronic colitis in mice. Front Cell Infect Microbiol. 2019;9:239.

34. Martens EC, Lowe EC, Chiang H, Pudlo NA, Wu M, McNulty NP, et al. Recognition and degradation of plant cell wall polysaccharides by two human gut symbionts. PLoS Biol. 2011;9:e1001221.

35. Pudlo NA, Urs K, Crawford R, Pirani A, Atherly T, Jimenez R, et al. Phenotypic and genomic diversification in complex carbohydrate-degrading human gut bacteria. mSystems. 2022;7:e0094721.

36. Lagkouvardos I, Pukall R, Abt B, Foesel BU, Meier-Kolthoff JP, Kumar N, et al. The mouse intestinal bacterial collection (miBC) provides host-specific insight into cultured diversity and functional potential of the gut microbiota. Nat Microbiol. 2016;1:16131.

37. Weldon L, Abolins S, Lenzi L, Bourne C, Riley EM, Viney M. The gut microbiota of wild mice. PLoS One. 2015;10:e0134643.

38. Segura Munoz RR, Quach T, Gomes-Neto JC, Xian Y, Pena PA, Weier S, et al. Stearidonic-enriched soybean oil modulates obesity, glucose metabolism, and fatty acid profiles independently of Akkermansia muciniphila. Mol Nutr Food Res. 2020;64:e2000162.

39. Bindels LB, Segura Munoz RR, Gomes-Neto JC, Mutemberezi V, Martinez I, Salazar $\mathrm{N}$, et al. Resistant starch can improve insulin sensitivity independently of the gut microbiota. Microbiome 2017;5:12.

40. Chen IA, Chu K, Palaniappan K, Pillay M, Ratner A, Huang J, et al. IMG/M v.5.0: an integrated data management and comparative analysis system for microbial genomes and microbiomes. Nucleic Acids Res. 2019;47:D666-D677.

41. Rozen S, Skaletsky H. Primer3 on the WWW for general users and for biologist programmers. Methods Mol Biol. 2000;132:365-86. 
42. Mukherjee S, Stamatis D, Bertsch J, Ovchinnikova G, Katta HY, Mojica A, et al. Genomes OnLine database (GOLD) v.7: Updates and new features. Nucleic Acids Res.2019;47:D649-D659.

43. Schneeberger M, Everard A, Gomez-Valades AG, Matamoros S, Ramirez S, Delzenne NM, et al. Akkermansia muciniphila inversely correlates with the onset of inflammation, altered adipose tissue metabolism and metabolic disorders during obesity in mice. Sci Rep. 2015;5:16643.

44. Gomes-Neto JC, Mantz S, Held K, Sinha R, Segura Munoz RR, Schmaltz R, et al. A real-time PCR assay for accurate quantification of the individual members of the Altered Schaedler Flora microbiota in gnotobiotic mice. J Microbiol Methods. 2017;135:52-62

45. Gomes-Neto JC, Kittana H, Mantz S, Segura Munoz RR, Schmaltz RJ, Bindels LB, et al. A gut pathobiont synergizes with the microbiota to instigate inflammatory disease marked by immunoreactivity against other symbionts but not itself. Sci Rep. 2017;7:17707.

46. Wingett SW, Andrews S. FastQ Screen: A tool for multi-genome mapping and quality control. F1000Res. 2018;7:1338.

47. Li H, Durbin R. Fast and accurate short read alignment with Burrows-Wheeler transform. Bioinformatics 2009;25:1754-60.

48. Garcia-Alcalde F, Okonechnikov K, Carbonell J, Cruz LM, Gotz S, Tarazona S, et al. Qualimap: evaluating next-generation sequencing alignment data. Bioinformatics 2012;28:2678-79.

49. Thomsen MCF, Hasman $H$, Westh $H$, Kaya $H$, Lund O. RUCS: rapid identification of PCR primers for unique core sequences. Bioinformatics 2017;33:3917-21.

50. Darling AC, Mau B, Blattner FR, Perna NT. Mauve: multiple alignment of conserved genomic sequence with rearrangements. Genome Res. 2004;14:1394-1403.

51. Kang DD, Li F, Kirton E, Thomas A, Egan R, An H, et al. MetaBAT 2: An adaptive binning algorithm for robust and efficient genome reconstruction from metagenome assemblies. PeerJ. 2019;7:e7359.

52. Genome [Internet] (2004). National Library of Medicine (US), National Center for Biotechnology Information: Bethesda (MD). https://www.ncbi.nlm.nih.gov/ genome/browse/\#!/prokaryotes/1218/

53. Genome [Internet] (2004). National Library of Medicine (US), National Center for Biotechnology Information: Bethesda (MD). https://www.ncbi.nlm.nih.gov/ genome/browse/\#!/prokaryotes/1598/

54. Beghini F, Mclver L, Blanco-Miguez A, Dubois L, Asnicar F, Maharjan S, et al. Integrating taxonomic, functional, and strain-level profiling of diverse microbial communities with bioBakery 3. Elife. 2021;10:e65088.

55. Asnicar F, Weingart G, Tickle TL, Huttenhower C, Segata N. Compact graphical representation of phylogenetic data and metadata with GraPhIAn. PeerJ 2015;3: e1029.

56. Varghese NJ, Mukherjee S, Ivanova N, Konstantinidis KT, Mavrommatis K, Kyrpides $\mathrm{NC}$, et al. Microbial species delineation using whole genome sequences. Nucleic Acids Res 2015:43:6761-71.

57. Mavromatis K, Chu K, Ivanova N, Hooper SD, Markowitz VM, Kyrpides NC. Gene context analysis in the Integrated Microbial Genomes (IMG) data management system. PLoS One 2009;4:e7979.

58. El-Gebali S, Mistry J, Bateman A, Eddy SR, Luciani A, Potter SC, et al. The Pfam protein families database in 2019. Nucleic Acids Res 2019;47:D427-D432.

59. The UniProt Consortium. The universal protein resource (UniProt). Nucleic Acids Res 2008:36:D190-195.

60. Obadia B, Guvener ZT, Zhang V, Ceja-Navarro JA, Brodie EL, Ja WW, et al. Probabilistic invasion underlies natural gut microbiome stability. Curr Biol 2017;27:1999-2006 e1998.

61. Meszena G, Gyllenberg M, Pasztor L, Metz JA. Competitive exclusion and limiting similarity: A unified theory. Theor Popul Biol. 2006;69:68-87.

62. Cavender-Bares J, Kozak KH, Fine PV, Kembel SW. The merging of community ecology and phylogenetic biology. Ecol Lett. 2009;12:693-715.

63. Tramontano $M$, Andrejev S, Pruteanu M, Klunemann M, Kuhn M, Galardini M, et al. Nutritional preferences of human gut bacteria reveal their metabolic idiosyncrasies. Nat Microbiol 2018;3:514-22.

64. Derrien $\mathrm{M}$, Vaughan EE, Plugge $\mathrm{CM}$, de Vos WM. Akkermansia muciniphila gen. nov., sp. nov., a human intestinal mucin-degrading bacterium. Int J Syst Evol Microbiol 2004;54:1469-1476.

65. Walker AW, Lawley TD. Therapeutic modulation of intestinal dysbiosis. Pharm Res 2013;69:75-86.

66. Livanos AE, Greiner TU, Vangay P, Pathmasiri W, Stewart D, McRitchie S, et al. Antibiotic-mediated gut microbiome perturbation accelerates development of type 1 diabetes in mice. Nat Microbiol 2016;1:16140.

67. Perez-Cobas AE, Gosalbes MJ, Friedrichs A, Knecht H, Artacho A, Eismann K, et al. Gut microbiota disturbance during antibiotic therapy: A multi-omic approach Gut 2013;62:1591-1601.

68. Adler PB, Hillerislambers J, Levine JM. A niche for neutrality. Ecol Lett. 2007;10:95-104
69. Levine JM, HilleRisLambers J. The importance of niches for the maintenance of species diversity. Nature 2009:461:254-57.

70. Forstner G. Signal transduction, packaging and secretion of mucins. Annu Rev Physiol. 1995;57:585-605.

71. Ottman N, Davids M, Suarez-Diez M, Boeren S, Schaap PJ, Martins Dos Santos VAP, et al. Genome-scale model and omics analysis of metabolic capacities of Akkermansia muciniphila reveal a preferential mucin-degrading lifestyle. Appl Environ Microbiol. 2017;83:e01014-17.

72. Duar RM, Frese SA, Lin XB, Fernando SC, Burkey TE, Tasseva G et al. Experimental evaluation of host adaptation of Lactobacillus reuteri to different vertebrate species. Appl Environ Microbiol. 2017;83:e00132-17.

73. Frese SA, Benson AK, Tannock GW, Loach DM, Kim J, Zhang M, et al. The evolution of host specialization in the vertebrate gut symbiont Lactobacillus reuteri. PLoS Genet. 2011;7:e1001314.

74. Rosshart SP, Vassallo BG, Angeletti D, Hutchinson DS, Morgan AP, Takeda K, et al. Wild mouse gut microbiota promotes host fitness and improves disease resistance. Cell 2017;171:1015-1028 e1013.

75. Karcher N, Nigro E, Puncochar M, Blanco-Miguez A, Ciciani M, Manghi $P$, et al Genomic diversity and ecology of human-associated Akkermansia species in the gut microbiome revealed by extensive metagenomic assembly. Genome Biol. 2021;22:209.

76. Rosshart SP, Herz J, Vassallo BG, Hunter A, Wall MK, Badger JH, et al. Laboratory mice born to wild mice have natural microbiota and model human immune responses. Science. 2019;365.

77. Mark Welch JL, Hasegawa Y, McNulty NP, Gordon J, Borisy GG. Spatial organization of a model 15-member human gut microbiota established in gnotobiotic mice. Proc Natl Acad Sci USA. 2017;114:E9105-E9114.

78. Whitaker WR, Shepherd ES, Sonnenburg JL. Tunable expression tools enable single-cell strain distinction in the gut microbiome. Cell 2017;169:538-546. e512.

79. Becken B, Davey L, Middleton DR, Mueller KD, Sharma A, Holmes ZC, et al. Genotypic and phenotypic diversity among human isolates of Akkermansia muciniphila. mBio. 2021;12:e00478-21.

80. Truong DT, Tett A, Pasolli E, Huttenhower C, Segata N. Microbial strain-level population structure and genetic diversity from metagenomes. Genome Res. 2017;27:626-38.

81. Faith JJ, Guruge JL, Charbonneau M, Subramanian S, Seedorf H, Goodman AL, et al. The long-term stability of the human gut microbiota. Science 2013;341:1237439.

82. Mehta RS, Abu-Ali GS, Drew DA, Lloyd-Price J, Subramanian A, Lochhead P, et al. Stability of the human faecal microbiome in a cohort of adult men. Nat Microbiol. 2018;3:347-355.

83. Ferretti P, Pasolli E, Tett A, Asnicar F, Gorfer V, Fedi S, et al. Mother-to-infant microbial transmission from different body sites shapes the developing infant gut microbiome. Cell Host Microbe. 2018:24:133-45 e135.

84. Korpela K, Costea P, Coelho LP, Kandels-Lewis S, Willemsen G, Boomsma DI, et al. Selective maternal seeding and environment shape the human gut microbiome. Genome Res. 2018;28:561-8.

85. Freitag TL, Hartikainen A, Jouhten $\mathrm{H}$, Sahl C, Meri S, Anttila VJ, et al. Minor effect of antibiotic pre-treatment on the engraftment of donor microbiota in fecal transplantation in mice. Front Microbiol. 2019;10:2685.

86. Ji SK, Yan H, Jiang T, Guo CY, Liu JJ, Dong SZ, et al. Preparing the gut with antibiotics enhances gut microbiota reprogramming efficiency by promoting xenomicrobiota colonization. Front Microbiol. 2017:8:1208.

87. Divya Ganeshan S, Hosseinidoust Z. Phage therapy with a focus on the human microbiota. Antibiotics (Basel). 2019;8:131.

88. Ramachandran G, Bikard D. Editing the microbiome the CRISPR way. Philos Trans R Soc Lond B Biol Sci. 2019:374:20180103.

\section{ACKNOWLEDGEMENTS}

This work was supported by a Young Investigator Grant in Probiotics from the Global Probiotics Council to AERT. JW is funded in part by Science Foundation Ireland Centre grant to APC Microbiome Ireland (APC/SFI/12/RC/2273_P2). We thank the staff of the Nebraska Gnotobiotic Mouse Program for their technical expertise and skillful animal husbandry. We also thank Dr. Daniel Peterson for kindly providing $B$. vulgatus $\mathrm{RJ} 2 \mathrm{H} 1$ for our experiments and Drs. Stephan Rosshart and Nicola Segata for helpful discussions. The funders had no role in the study design, data collection and analysis, decision to publish, or preparation of the manuscript.

\section{AUTHOR CONTRIBUTIONS}

RRSM, JW, and AERT conceived the overall project objectives, designed experiments, interpreted data, and wrote the manuscript with contributions from all authors. 
RRSM, SM, IM, RJS conducted animal studies, collected samples, and performed qPCR and $16 \mathrm{~S}$ rRNA gene sequencing analyses. FL performed metagenomics analyses. NAP and $\mathrm{KU}$ performed $B$. vulgatus carbohydrate utilization analyses with support and guidance from ECM. AERT and JW secured funding for the project.

\section{COMPETING INTERESTS}

The authors declare no competing interests.

\section{ADDITIONAL INFORMATION}

Supplementary information The online version contains supplementary material available at https://doi.org/10.1038/s41396-022-01208-9.

Correspondence and requests for materials should be addressed to Jens Walter or Amanda E. Ramer-Tait.

Reprints and permission information is available at http://www.nature.com/ reprints
Publisher's note Springer Nature remains neutral with regard to jurisdictional claims in published maps and institutional affiliations.

\section{(c) (i)}

Open Access This article is licensed under a Creative Commons Attribution 4.0 International License, which permits use, sharing, adaptation, distribution and reproduction in any medium or format, as long as you give appropriate credit to the original author(s) and the source, provide a link to the Creative Commons license, and indicate if changes were made. The images or other third party material in this article are included in the article's Creative Commons license, unless indicated otherwise in a credit line to the material. If material is not included in the article's Creative Commons license and your intended use is not permitted by statutory regulation or exceeds the permitted use, you will need to obtain permission directly from the copyright holder. To view a copy of this license, visit http://creativecommons. org/licenses/by/4.0/.

(c) The Author(s) 2022, corrected publication 2022 